\title{
Extraction of Transverse Momentum Distributions from semi-inclusive DIS and Drell-Yan data.
}

\author{
Filippo Delcarro* \\ Università di Pavia and INFN, via Bassi 6,I-27100 Pavia \\ E-mail: filippo.delcarro@pv.infn.it
}

\section{Alessandro Bacchetta \\ Università di Pavia and INFN, via Bassi 6,I-27100 Pavia \\ E-mail: alessandro.bacchetta@unipv.it}

\section{Cristian Pisano}

Università di Cagliari and INFN, Cittadella Universitaria, I-09042 Monserrato (CA)

E-mail: cristian.pisanodca.infn.it

\section{Marco Radici}

INFN, Sezione di Pavia, via Bassi 6,I-27100 Pavia

E-mail: marco.radici@pv.infn.it

\section{Andrea Signori}

Theory Center, Thomas Jefferson National Accelerator Facility, 12000 Jefferson Avenue,

Newport News, VA 23606, USA

E-mail: asignoriejlab.org

\begin{abstract}
We present a first attempt at a global extraction of unpolarized partonic transverse momentum dependent (TMD) distributions and fragmentation functions from a simultaneous fit of data measured in semi-inclusive deep-inelastic scattering, Drell-Yan and $Z$ boson production. This analysis is performed in the low transverse momentum region, at leading order in perturbative QCD, implementing TMD evolution at NLL accuracy. We test the agreement between our results and the recently published COMPASS data for muon-deuteron SIDIS multiplicities, and discuss their inclusion in a future global fit with a higher accuracy.
\end{abstract}

XXVI International Workshop on Deep-Inelastic Scattering and Related Subjects (DIS2018) 16-20 April 2018

Kobe, Japan

${ }^{*}$ Speaker. 


\section{Introduction}

Parton distribution functions describe the internal structure of the nucleon in terms of its elementary constituents, quarks and gluons. Transverse momentum dependent distributions and fragmentations functions (TMDs) carry fundamental information on the intrinsic motion of partons and the correlation between the nucleons spins and momenta, including however also the dependence on transverse momentum components $k_{\perp}^{2}$ and thus providing a full three-dimensional picture of hadrons in momentum space.

In this contribution to the proceedings, based on [1] to which we refer for a more detailed discussion, we focus only on the unpolarized TMD PDF $f_{1}^{q}\left(x, k_{\perp}^{2}, Q^{2}\right)$ and the unpolarized TMD FF $D_{1}^{q \rightarrow h}\left(z, P_{h T}^{2}, Q^{2}\right)$, with a flavor independent analysis. We take into consideration three processes: semi-inclusive DIS, and Drell-Yan processes (DY), both with the production of virtual photons and $Z$ bosons. Many observables in hadronic hard scattering experiments are related to PDFs and FFs, in a way specified by factorization theorems (see, e.g., Refs. [2, 3]). These theorems also illustrate the universality of PDFs and FFs (i.e., the fact that they are the same in different processes) and their evolution equations. Availability of measurements of different processes in different experiments makes it possible to test the reliability of factorization theorems and extract PDFs and FFs through so-called global fits.

\section{Formalism}

In one-particle SIDIS, a lepton $\ell$ with momentum $l$ scatters off a hadron target $N$ with mass $M$ and momentum $P$. In the final state, the scattered lepton momentum $l^{\prime}$ is measured together with one hadron $h$ with mass $M_{h}$ and momentum $P_{h}$. The available data refer to SIDIS hadron multiplicities; in the single-photon-exchange approximation, the multiplicities can be written as (see Ref. [4] for details):

$$
m_{N}^{h}\left(x, z,\left|\boldsymbol{P}_{h T}\right|, Q^{2}\right)=\frac{d \sigma_{N}^{h} /\left(d x d z d\left|\boldsymbol{P}_{h T}\right| d Q^{2}\right)}{d \sigma_{D I S} /\left(d x d Q^{2}\right)} \simeq \frac{2 \pi\left|\boldsymbol{P}_{h T}\right| F_{U U, T}\left(x, z, \boldsymbol{P}_{h T}^{2}, Q^{2}\right)}{F_{T}\left(x, Q^{2}\right)}
$$

where $x, y, z$ and $\gamma$ are the usual kinematic variables, $\boldsymbol{P}_{h T}$ is the component of $\boldsymbol{P}_{h}$ transverse to $\left.\boldsymbol{q}, Q^{2}=-q^{2}=-\left(l-l^{\prime}\right)^{2}\right)$ and $\varepsilon$ is the polarization ratio of the virtual photon.

The semi-inclusive cross section can be factorized in terms of TMDs only in the kinematic limits $M^{2} \ll Q^{2}$ and $\boldsymbol{P}_{T}^{2} \ll Q^{2}$. Moreover, in the present analysis, we will take into account all powers of the form $\alpha_{S}^{n} L^{2 n} \approx 1$ (Leading Logarithms -LL) and $\alpha_{S}^{n} L^{n} \approx 1$ (Next-to-Leading Logarithms NLL). With this accuracy, only the contribution $F_{U U, T}$ is relevant in our study.

In our study we neglect the terms $\mathscr{H}_{U U, T}$, denoting the NLO hard scattering part, and $Y_{U U, T}$, introduced to ensure a matching to the perturbative fixed-order calculations at higher transverse momenta. In order to apply TMD evolution equations, we need to calculate the Fourier transform of the part of $F_{U U, T}$ involving TMDs. The structure function thus reduces to

$$
F_{U U, T}\left(x, z, \boldsymbol{P}_{h T}^{2}, Q^{2}\right) \approx 2 \pi \sum_{a} \int_{0}^{\infty} d \xi_{T} \xi_{T} J_{0}\left(\xi_{T}\left|\boldsymbol{P}_{h T}\right| / z\right) \tilde{f}_{1}^{a}\left(x, \xi_{T}^{2} ; Q^{2}\right) \tilde{D}_{1}^{a \nrightarrow h}\left(z, \xi_{T}^{2} ; Q^{2}\right)
$$


A similar discussion can be made for a Drell-Yan process, where two hadrons $A$ and $B$ with momenta $P_{A}$ and $P_{B}$ collide at a center-of-mass energy squared $s=\left(P_{A}+P_{B}\right)^{2}$ and produce a virtual photon or a $Z$ boson plus hadrons. The differential cross sections can be analyzed in terms of structure functions $F_{U U}^{1}$ and $F_{U U}^{2}[5,6]$. Similarly to the SIDIS case, in the kinematic limit $q_{T}^{2} \ll Q^{2}$ and not including the hadron masses the structure function $F_{U U}^{2}$ can be neglected. With the choices discussed for the SIDIS case, the structure function $F_{U U}^{1}$ can be expressed as a Fourier transform:

$$
F_{U U}^{1}\left(x_{A}, x_{B}, \boldsymbol{q}_{T}^{2}, Q^{2}\right)=\sum_{a} \int_{0}^{\infty} \frac{d \xi_{T}}{2 \pi} \xi_{T} J_{0}\left(\xi_{T}\left|\boldsymbol{q}_{T}\right|\right) \tilde{f}_{1}^{a}\left(x_{A}, \xi_{T} ; \mu^{2}\right) \tilde{f}_{1}^{\bar{a}}\left(x_{B}, \xi_{T} ; \mu^{2}\right) .
$$

In order to include measurement taken at different energy scales, we implement the formalism for TMD evolution in our model, containing the connection between TMDs at different values of the hard scale of the process. Following the formalism of Refs. [3, 7], the evolved unpolarized TMD distribution at LO in configuration space for a parton flavor $a$ at a scale $Q^{2}$ can be written as

$$
\widetilde{f}_{1}^{a}\left(x, \xi_{T}^{2} ; Q^{2}\right)=f_{1}^{a}\left(x ; \mu_{b}^{2}\right) e^{S\left(\mu_{b}^{2}, Q^{2}\right)} \cdot e^{g_{K}\left(\xi_{T}\right) \ln \left(Q^{2} / Q_{0}^{2}\right)} \widetilde{f}_{1 \mathrm{NP}}^{a}\left(x, \xi_{T}^{2}\right)
$$

where $f_{1}^{a}\left(x ; \mu_{b}^{2}\right)$ is the usual collinear distribution function, evaluated at the initial energy scale $\mu_{b}$. According to Refs. [8, 9, 10], for the nonperturbative Sudakov factor we make the traditional choice $g_{K}\left(\xi_{T}\right)=-g_{2} \xi_{T}^{2} / 2$ with $g_{2}$ a free parameter. Finally, we parametrize the intrinsic nonperturbative parts of the TMDs as the normalized linear combination of a Gaussian and a weighted Gaussian:

$$
\begin{aligned}
\widetilde{f}_{1 \mathrm{NP}}^{a}\left(x, \xi_{T}^{2}\right) & =\frac{1}{2 \pi} e^{-g_{1 a} \frac{\xi_{T}^{2}}{4}}\left(1-\frac{\lambda g_{1 a}^{2}}{1+\lambda g_{1 a}} \cdot \frac{\xi_{T}^{2}}{4}\right) \\
\widetilde{D}_{1 \mathrm{NP}}^{a \rightarrow h}\left(z, \xi_{T}^{2}\right) & =\frac{g_{3 a \rightarrow h} e^{-g_{3 a \rightarrow h} \frac{\xi_{T}^{2}}{4}}+\left(\lambda_{F} / z^{2}\right) g_{4 a \rightarrow h}^{2}\left(1-g_{4 a \rightarrow h} \frac{\xi_{T}^{2}}{4}\right) e^{-g_{4 a \rightarrow h} \frac{\xi_{T}^{2}}{4}}}{2 \pi z^{2}\left(g_{3 a \rightarrow h}+\left(\lambda_{F} / z^{2}\right) g_{3 a \rightarrow h}\right)} .
\end{aligned}
$$

Based on the analyses of Refs. [11, 12], we defined the Gaussian width of the TMDs to be dependent on their fractional momentum:

$$
g_{1}(x)=N_{1} \frac{(1-x)^{\alpha} x^{\sigma}}{(1-\hat{x})^{\alpha} \hat{x}^{\sigma}}, \quad g_{3,4}(z)=N_{3,4} \frac{\left(z^{\beta}+\delta\right)(1-z)^{\gamma}}{\left(\hat{z}^{\beta}+\delta\right)(1-\hat{z})^{\gamma}}
$$

where $\alpha, \sigma, N_{1} \equiv g_{1}(\hat{x})$ with $\hat{x}=0.1$ and $\beta, \gamma, \delta, N_{3,4} \equiv g_{3,4}(\hat{z})$ with $\hat{z}=0.5$ are free parameters. In total we need 11 free parameters to describe our model, defined more in depth in [1].

\section{Data analysis and results}

In our work we included a wide range of measurements taken from semi-inclusive DIS, DrellYan at low energy and $Z$ boson production.

To avoid issues relative to known errors in the normalization of Compass data, we divided every data point by the value of the first point of their bin, excluding them from the d.o.f counting. The application of the TMD formalism to SIDIS depends on the capability of identifying the current fragmentation region, we identify it by operating a cut on $z$ only, namely $0.2<z<0.74$. Another 
requirement for the applicability is the presence of two separate scales in the process, $Q^{2}$ and $P_{h T}^{2}$, which should satisfy the condition $P_{h T}^{2} / z^{2} \ll Q^{2}$. We implement this condition by imposing $Q^{2}>$ $1.4 \mathrm{GeV}^{2}$ and restricting our fit to the small transverse momentum region. With those kinematic cuts we have 6252 points from COMPASS , 1514 from HERMES, 203 from Drell-Yan experiments and another 90 from $\mathrm{Z}$ boson production, for a total of 8059 data points.

Our fit is based on the replica methodology, a Monte Carlo approach that consists in creating $\mathscr{M}$ replicas of the data points, by shifting independently each data point $i$ by a Gaussian noise with the same variance as the measurement. A minimization procedure is applied to each replica separately, using MinUiT.

The overall quality of our fit is good, with a global $\chi^{2} /$ d.o.f. $=1.55 \pm 0.05$. Uncertainties are computed as the $68 \%$ confidence level (C.L.) from the replica methodology. The number of degrees of freedom (d.o.f.) is given by the number of data points analyzed, reduced by the number of free parameters (i.e. 11 free parameters) and the number of points used as normalization.

With the adopted kinematic cuts, we included regions where TMD factorization could be questioned, but we checked that our results describe very well the regions where TMD factorization is supposed to hold. The $\chi^{2} /$ d.o.f. can be improved up to 1.02 restricting the kinematic cuts, without changing the parameters. In addition, we tested the stability of our distribution shape agianst different possible choices for collinear pdf distributions, alternative kinematic cuts and including normalization also for HERMES .

\section{Preliminary analysis of recently published SIDIS COMPASS data}

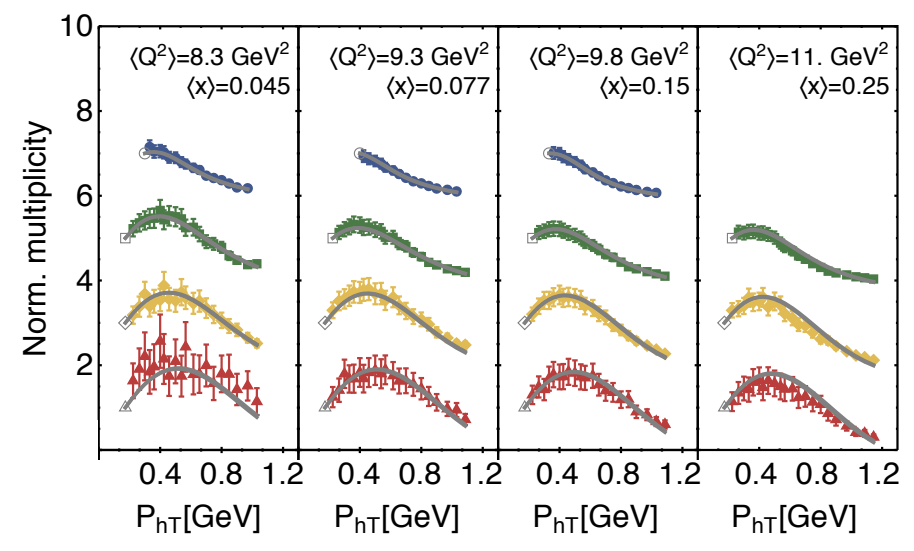

Figure 1: Updated COMPASS multiplicities [13], as a function of the transverse momentum of the detected hadron $P_{h T}$ at different $\langle x\rangle,\langle z\rangle,\left\langle Q^{2}\right\rangle$ bins. Grey bands represent the $68 \%$ confidence level of the theoretical curves obtained from the minimization of 50 data replicas. Each $\langle z\rangle$ bin has been shifted for clarity by an offset.

Recently COMPASS collaboration has published a new semi-inclusive measurement of charged hadron multiplicities from muon-deuteron DIS [13]. Comparing this dataset to the one used in our previously discussed analysis [14], we observe that they cover a wider kinematical range, reaching in $\mathrm{x}$ up to 0.4 instead of 0.12 , in $\mathrm{Q}^{2}$ up to $81 \mathrm{GeV}^{2}$ instead of $10 \mathrm{GeV}^{2}$ and in $\mathrm{P}_{h T}^{2}$ up to $3 \mathrm{GeV}^{2}$ instead of about $1 \mathrm{GeV}^{2}$. Even if the data points are reduced in quantity they have a much higher 
statistics, leading to a significantly decreased systematic uncertainties on the normalization of the $\mathrm{P}_{h T}^{2}$-integrated multiplicities. This feature could be an important improvement for our analysis, considering that at the moment it seems to be necessary to normalize each $\mathrm{x}, \mathrm{z}, \mathrm{Q}^{2}$ bin of CoMPASS data.

We conducted a preliminary study of this new data set to test its agreement with the result obtained in our previous global fit. As a first step we compared the recent COMPASS data with the theoretical curves obtained from the 200 groups of parameters extracted from the minimization of the already discussed data replicas. In this case we obtained a $\chi^{2}$ value of 1.91 if we considered only the COMPASS measurements, and a value of 2.01 if we also included Drell-Yan and $Z$ boson production data. These initial results suggest that the parameters obtained in the previous fit are able to describe also the new one, as can be observed in Fig. 1, even if not as accurately as before. After this initial exploration we repeated our global minimization on 50 replicas of the new data set. After minimizing we obtained a parameter space similar to the published one, able to describe the experimental measurements quite accurately ( $\chi^{2}=1.71$ with 3922 d.o.f.). Finally we tested the necessity of the bin normalization for the improved data. A preliminary comparison without minimization and using the parameters obtained from previous fits showed a worse agreement with the measurements. Even repeating the minimization process at the moment we are not able to achieve the results of our previous analysis. There are different issues that could be the cause of this behaviour, we noticed that including this data our results became more sensitive to the value of $z$, producing notably different theoretical curves if we choose the starting or the mid value of a $z$ bin. This sensitivity can be explained observing how the shape of the FF changes in relation to $z$ and considering that the $z$ bin are now twice larger than before; integrating the multiplicities on the entire $z$ bin range instead of evaluating them for the average value could solve this issue.

We observed also that the updated data seem to be less stable with regards to the kinematic cuts applied, and it could be necessary to resize the kinematic range in order to find an area with greater stability.

Finally, we are implementing in our future analysis higher order corrections to exclude that discrepancies between our model and the data could come from an incomplete description of the process.

\section{Conclusions}

In this work we demonstrated for the first time that it is possible to perform a simultaneous fit of unpolarized TMD PDFs and FFs to data of SIDIS, Drell-Yan and $Z$ boson production at small transverse momentum collected by different experiments.

Using a replica methodology we extracted unpolarized TMDs using 8059 data points with 11 free parameters, obtaining an average $\chi^{2} /$ d.o.f. of $1.55 \pm 0.05$. Most of the discrepancies between data and theory come from the normalization and not from the transverse momentum shape.

Preliminary studies on the recently published COMPASS sets of multiplicities suggest that they are compatible with our results, however further consideration on the normalization and the description of the $z$ dependence are necessary for a future improved global fit. 


\section{Acknowledgments}

This research has received funding from the European Research Council (ERC) under the European Union's Horizon 2020 research and innovation programme (grant agreement No. 647981, 3DSPIN).

AS acknowledges support from U.S. Department of Energy contract DE-AC05-06OR23177, under which Jefferson Science Associates, LLC, manages and operates Jefferson Lab.

Preprint submitted as JLAB-THY-18-2758.

\section{References}

[1] A. Bacchetta, F. Delcarro, C. Pisano, M. Radici and A. Signori, Extraction of partonic transverse momentum distributions from semi-inclusive deep-inelastic scattering, drell-yan and z-boson production, Journal of High Energy Physics 2017 (2017) 81.

[2] J. C. Collins, D. E. Soper and G. F. Sterman, Factorization of Hard Processes in QCD, Adv. Ser. Direct. High Energy Phys. 5 (1989) 1 [hep-ph/ 0409313$].$

[3] J. Collins, Foundations of perturbative QCD. Cambridge University Press, 2013.

[4] A. Bacchetta, M. Diehl, K. Goeke, A. Metz, P. J. Mulders and M. Schlegel, Semi-inclusive deep inelastic scattering at small transverse momentum, JHEP 02 (2007) 093 [hep-ph/ 0611265 ].

[5] D. Boer and W. Vogelsang, Drell-Yan lepton angular distribution at small transverse momentum, Phys. Rev. D74 (2006) 014004 [hep-ph/ 0604177 ].

[6] S. Arnold, A. Metz and M. Schlegel, Dilepton production from polarized hadron hadron collisions, Phys. Rev. D79 (2009) 034005 [0809.2262].

[7] S. M. Aybat and T. C. Rogers, TMD Parton Distribution and Fragmentation Functions with QCD Evolution, Phys. Rev. D83 (2011) 114042 [1101.5057].

[8] P. M. Nadolsky, D. R. Stump and C. P. Yuan, Semiinclusive hadron production at HERA: The Effect of QCD gluon resummation, Phys. Rev. D61 (2000) 014003 [hep-ph/9906280].

[9] F. Landry, R. Brock, P. M. Nadolsky and C. P. Yuan, Tevatron Run-1 Z boson data and Collins-Soper-Sterman resummation formalism, Phys. Rev. D67 (2003) 073016 [hep-ph/0212159].

[10] A. V. Konychev and P. M. Nadolsky, Universality of the Collins-Soper-Sterman nonperturbative function in gauge boson production, Phys. Lett. B633 (2006) 710 [hep-ph / 0506225$].$

[11] A. Signori, A. Bacchetta, M. Radici and G. Schnell, Investigations into the flavor dependence of partonic transverse momentum, JHEP 11 (2013) 194 [1309.3507].

[12] A. Bacchetta, M. G. Echevarria, P. J. G. Mulders, M. Radici and A. Signori, Effects of TMD evolution and partonic flavor on $e^{+} e^{-}$annihilation into hadrons, JHEP 11 (2015) 076 [1508.00402].

[13] COMPASS Collaboration collaboration, M. Aghasyan, M. G. Alexeev, G. D. Alexeev, A. Amoroso, V. Andrieux, N. V. Anfimov et al., Transverse-momentum-dependent multiplicities of charged hadrons in muon-deuteron deep inelastic scattering, Phys. Rev. D 97 (2018) 032006.

[14] COMPASS collaboration, C. Adolph et al., Hadron Transverse Momentum Distributions in Muon Deep Inelastic Scattering at 160 GeV/c, Eur. Phys. J. C73 (2013) 2531 [1305 . 7317]. 